\title{
Crack growth resistance of shape memory alloys by means of a cohesive zone model
}

\author{
Yuval Freed* and Leslie Banks-Sills \\ The Dreszer Fracture Mechanics Laboratory \\ Department of Solid Mechanics, Materials and Systems \\ The Fleischman Faculty of Engineering \\ Tel Aviv University \\ 69978 Ramat Aviv, ISRAEL
}

\begin{abstract}
Crack growth resistance of shape memory alloys is dominated by the transformation zone in the vicinity of the crack tip. In this study, the transformation toughening behavior of a slowly propagating crack in a shape memory alloy under plane strain conditions and mode I deformation is numerically investigated. A small scale transformation zone is assumed. A cohesive zone model is implemented to simulate crack growth within a finite element scheme. Resistance curves are obtained for a range of parameters that specify the cohesive traction-separation constitutive law. It is found that the choice of the cohesive strength $t_{0}$ has a great influence on the toughening behavior of the material. Moreover, the reversibility of the transformation can significantly reduce the toughening of the alloy. The shape of the initial transformation zone, as well as that of a growing crack is determined. The effect of the Young's moduli ratio of the martensite and austenite phases is examined.
\end{abstract}

Key words: Phase transformation, Fracture mechanisms, Finite elements, Shape memory alloys.

\section{Introduction}

Shape memory alloys (SMAs) have received much attention in recent years, especially as a result of their various applications in smart structures, actuators, medical devices, space and aeronautics. These materials exhibit extremely

* Corresponding author: yuval@eng.tau.ac.il, tel: +972-3-640-5581, fax: +972-3640-7617 


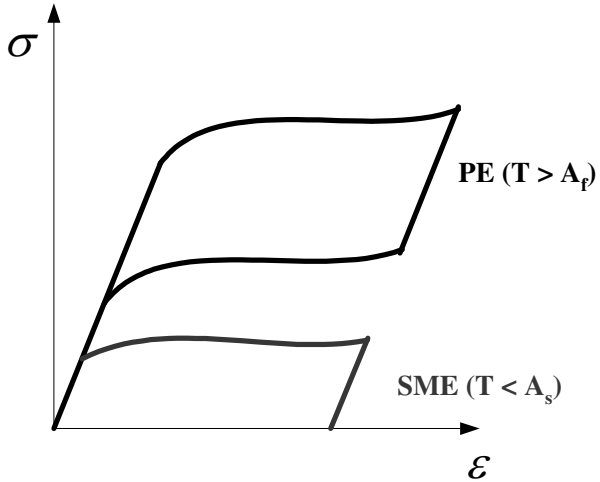

(a)

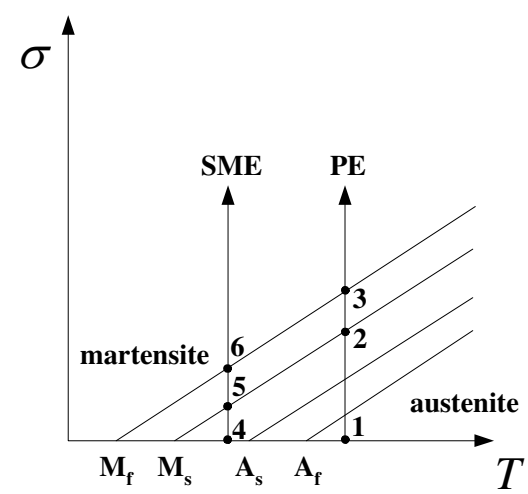

(b)

Fig. 1. (a) Typical stress-strain curves of a SMA. (b) Typical stress-temperature curves. If $T>A_{f}$ (point 1 ), $\mathrm{PE}$ occurs as a result of a stress induced transformation (points 2 to 3 ). If $T<A_{s}$ (point 4), SME resulting from a stress induced transformation (points 5 to 6 ) is achieved.

large, recoverable strains (of the order of 10\%), resulting from transformation between austenitic and martensitic phases. This transformation may be induced by a change, either in the applied stress (stress-induced transformation), the temperature (temperature-induced transformation), or a combination of the two.

From a macroscopic point of view, one may separate the observable behavior of SMAs into two major phenomena. The first one is known as pseudo-elasticity $(\mathrm{PE})$, in which nonlinear elastic behavior is observed (see Fig. 1a). Here, very large strains upon loading occur, but full recovery is achieved in a hysteresis loop upon unloading. When an SMA experiences the shape memory effect (SME), it exhibits a large residual strain after loading and unloading as illustrated in Fig. 1a. This strain may be fully recovered simply by raising the temperature of the body.

The relationship between the stress and temperature is presented in Fig. 1b. The various temperatures denoted along the abscissa are $M_{f}, M_{s}, A_{s}$, and $A_{f}$, respectively, the martensite final and start temperatures, and the austenite start and final temperatures. It should be noted that above the curve denoted with $M_{f}$, the material is fully martensitic; whereas, below the curve denoted by $A_{f}$, it is fully austenitic. For further discussion on this topic, see Patoor et al. (2006). In addition to the phase transformation associated with a temperature change, the transformation may be stress induced. In this case, the phase changes from austenite to martensite upon tensile loading, with constant temperature. This temperature dictates whether shape memory effect or pseudo-elasticity is taking place. If the temperature $T>A_{f}$ with $\sigma=0$ (point 1 in Fig. 1b), PE occurs. Upon loading, the austenite transforms to martensite (points 2 to 3); once unloading the body, full recovery is observed (see Fig. 1a), since austenite is the only stable phase at this temperature. If $T<A_{s}$ (point 4 in Fig. 1b), SME occurs. Similar to PE, the austenite 
transforms to martensite (points 5 to 6 ); upon unloading, there is no reverse transformation, since austenite is not a stable phase at this temperature, and residual strains are observed as exhibited in Fig. 1a. If $A_{s}<T<A_{f}$, a reverse transformation occurs upon unloading, but still some residual strains are observed. For both stress or temperature induced martensite, a hysteresis loop is observed in the stress-strain curve. This loop may be explained using the material stress-temperature relations; for the case of stress induced martensite, the forward and reverse transformation occur in different ranges of stresses. Hence, the recovery followed by the reverse transformation is on a path different from that of the loading path.

Different applications of shape memory alloys may be found in various fields, such as civil engineering (see for example, Janke et al., 2005, Song et al., 2006), medical devices (Morgan, 2004), actuators (Luo and Salvia, 2004), and in general engineering (Razov, 2004). This results from their relatively good mechanical properties (high strength, corrosion resistance), bio-compatibility, and a damping capacity which is far greater than that of standard materials. However, there are also several limitations. These alloys are still relatively expensive to manufacture with respect to ordinary metals such as steel and aluminum. Furthermore, they have poor fatigue properties which have been found to be two orders of magnitude lower than those of these metals (see Patoor et al., 2006). Consequently, SMA devices in which the pseudoelasticity or the shape memory effect are repeated may be designed for only a relatively small number of transformation cycles.

With the increasing use of SMAs in recent years, it is important to investigate the effect of cracks. Theoretically, the stress field near the crack tip is unbounded. Hence, a stress-induced transformation occurs, and the martensite phase is expected to appear in the neighborhood of the crack tip, from the very first loading step. In that case, the crack tip region is not governed by the far field stress, but rather by the crack tip stress field. This behavior implies transformation toughening or softening.

The failure mechanisms of SMAs have been studied in recent years. However, most investigations are experimental, as reviewed by Wilkes and Liew (2000). In a recent paper by Robertson and Ritchie (2007), the fracture toughening behavior of an SMA tube was investigated experimentally. Crack resistance curves were obtained which exhibit significant toughening. Only a few theoretical studies on the failure of SMAs have been published. Among them, Yi and Gao (2000) and Yi et al. (2001) examined the transformation toughening of an SMA in mode I and mixed modes. In both cases, the constitutive model presented by Sun and Hwang (1993a, 1993b) was used in the analyses following closely that of McMeeking and Evans (1982) for zirconia. The Eshelby equivalent inclusion method was used to obtain the R-curve for several loading cases; no reverse transformation process was considered. An earlier model of Sun et 
al. (1991) was used by Stam and van der Giessen (1995) to examine the effect of partial and fully reversed transformations on crack growth resistance in the regime of pseudo-elasticity. In their study, they observed substantial transformation toughening, and found that the reverse transformation can significantly reduce the resistance increase. Finally, Yan et al. (2002) examined the effect of transformation volume contraction on the toughening of pseudoelastic shape memory alloys focusing specifically on the case in which there is a residual volumetric transformation strain upon unloading. This case is reminiscent of the well known stress-induced transformation phenomenon in zirconia. However, unlike zirconia, this transformation is characterized by a negative volumetric change; hence, transformation softening occurs. In order to obtain the change in the stress intensity factor, both Stam and van der Giessen (1995) and Yan et al. (2002) used a solution presented by Hutchinson (1974) for two spots of plastically deformed material that are located symmetrically with respect to the crack line. This solution is suitable for the irreversible supercritical case, when the transformation is discontinuous, with complete transformation in the process zone and no transformation outside of it (see Budiansky et al., 1983, for further information). Moreover, since both Yi and Gao (2000) and Yi et al. (2001) used the Eshelby equivalent inclusion method to obtain the change of the stress intensity factors, similar to the Hutchinson (1974) solution, it is valid only for an irreversible supercritical transformation; however, SMAs exhibit a nonuniform distribution of the transformation strain (subcritical transformation). Hence, none of the above investigations fully characterize the behavior of shape memory alloys resulting from crack propagation.

The first objective of this study is to obtain the transformation toughening behavior of shape memory alloys. The energy dissipation caused by the high stresses in the transformation zone in the vicinity of the crack tip plays a major role in the transformation toughening of the alloy. Since this dissipation depends upon crack advance, the outcome of this approach is a crack growth resistance curve.

It may be pointed out that the yield stress of shape memory alloys is far greater than the transformation stresses. For example, the yield stress of Nitinol was found to be 1,058 MPa (see McKelvey and Ritchie, 2001). This value may be one to several orders of magnitude larger than the effective stress which induces a martensite transformation. Hence, the plastic zone will be well within the martensite transformation region and may be neglected.

In conventional linear elastic fracture mechanics, the crack tip is characterized by a single macroscopic parameter such as the stress intensity factor $\left(K_{I}, K_{I I}\right.$ or $K_{I I I}$ ) or a corresponding energy release rate. This approach is commonly used, but it cannot account for the case in which the crack growth resistance increases with crack length, as a result of energy dissipation. To solve this problem, a cohesive zone model is adopted. This model was first proposed by 
Barenblatt $(1959,1962)$ and Dugdale $(1960)$ as an alternative approach to singular crack tip behavior. With this concept, the stresses ahead of the crack tip are bounded, and a traction-separation law is proposed to describe the fracture process. Once the energy in the process zone approaches the critical Griffith energy release rate, the crack propagates.

Although this concept was investigated extensively by many researchers, most of these studies differ from each other by the specific traction-separation law. For example, Needleman (1987) used a potential function to obtain a polynomial law, and investigated particle-matrix decohesion. Tvergaard and Hutchinson (1992) examined the crack growth resistance in elasto-plastic materials using a law in which the traction initially increases linearly with crack opening displacement (COD) reaching a region in which the traction is a constant for increasing COD. In the last stage of separation, the traction decreases linearly. On the other hand, Geubelle and Baylor (1998) used a bilinear traction separation law and examined a delamination caused by low velocity impact.

It is commonly assumed that cohesive zone models may be described by two independent parameters, which may be any two out of three parameters, namely, cohesive energy $\Gamma_{c}$, cohesive strength $t_{0}$ and the critical separation length $\delta_{c}$. Consequently, the second objective of this study is to examine the relation between these parameters and the crack growth resistance. Several other related issues, such as the shape and size of the initial transformation zone, the effect of the Young's moduli ratio on crack resistance, and the contribution of reverse transformation are explored.

This paper is organized as follows. First, the chosen constitutive model is described in Section 2. In Section 3, it is used to obtain the approximate size and shape of the initial transformation zones. Then, a one-dimensional cohesive interface element for a bilinear traction-separation law is described in Section 4. Finally, in Section 5, numerical results are presented and discussed. Both forward and reversed transformations are considered.

\section{Constitutive modeling of monolithic shape memory alloy}

The macroscopic mechanical behavior of SMAs is usually modeled from either a phenomenological or a micromechanical point of view. Most of the existing three-dimensional phenomenological models were derived in the spirit of plasticity or viscoplasticity, with an internal variable such as the martensite fraction. Among these models, the most acknowledged work is that of Boyd and Lagoudas (1996a, 1996b). Later, Souza et al. (1998) and Auricchio and Petrini (2002, 2004a, 2004b) introduced models which agree well with experimental observations, in particular multi-axial tests. The great advantage of 
these types of phenomenological models is that they can be easily implemented in numerical schemes, such as finite element analysis.

Other researchers, such as Patoor et al. (1996) and Lu and Weng (1997), used a micromechanics approach. The crystallographic behavior within the material was closely followed. These models are obviously much more complicated than the phenomenological models, but they appear to offer the best approach to describe the three-dimensional behavior of SMAs. Nevertheless, since these models cannot be implemented in a straight forward manner into a numerical scheme, a phenomenological model is used in this investigation.

In this study, the model proposed by Panoskaltsis et al. (2004) is employed. This phenomenological model is based on a general framework for the derivation of the general three-dimensional thermomechanical governing equations for shape memory alloys. The framework is a combination between generalized plasticity theory and the theory of continuum damage mechanics. Moreover, it is possible to describe different material moduli for austenite and martinsite.

As in many other models, this model employs only one internal variable, namely the scalar $\xi$ which describes the martensite fraction, so that $0 \leq \xi \leq 1$. The general form of the transformation surface is taken as a von Mises type surface, and given by

$$
F(\boldsymbol{\sigma}, T)=\sqrt{3 J_{2}}-C T
$$

where $\boldsymbol{\sigma}$ is the Cauchy stress tensor, $J_{2}=\boldsymbol{s}: \boldsymbol{s} / 2$ is the second invariant of the deviatoric stress tensor $\boldsymbol{s}, C$ is a material property, $T$ is the current temperature and the loading surface $F(\boldsymbol{\sigma}, T)$ is constant. For the austenite to martensite transformation, the surface is given by

$$
F_{M}(\boldsymbol{\sigma}, T)=\sqrt{3 J_{2}}-C_{M} T
$$

where $C_{M}$ is the slope of the one-dimensional stress-temperature diagram for martensite behavior (see Fig. 1b). By introducing $F_{M_{s}}$ and $F_{M_{f}}$ as the loading surfaces at the beginning and the end of the transformation, it is possible to write that

$$
\begin{aligned}
& F_{M_{s}}(\boldsymbol{\sigma}, T)=\sqrt{3 J_{2}}-C_{M}\left(T-M_{s}\right), \\
& F_{M_{f}}(\boldsymbol{\sigma}, T)=\sqrt{3 J_{2}}-C_{M}\left(T-M_{f}\right),
\end{aligned}
$$

where $M_{s}$ and $M_{f}$ represent the martensite start and finish temperatures, 
respectively. It should be noted that the product $F_{M_{s}} F_{M_{f}}$ is negative only during the austenite to martensite phase transformation. Hence, the expression for evolution of the martensite fraction during the forward transformation is given by

$$
\dot{\xi}=-\frac{\left\langle-F_{M_{s}} F_{M_{f}}\right\rangle}{\left|F_{M_{s}} F_{M_{f}}\right|} \frac{1-\xi}{F_{M_{f}}}\left\langle\dot{F}_{M}\right\rangle,
$$

where $\langle\cdot\rangle$ is the Macauley bracket, defined as $\langle x\rangle=(|x|+x) / 2$ and the dot above a quantity represents its increment. Evolution of the transformation strain tensor, or the so called 'flow rule', is given as

$$
\dot{\boldsymbol{\epsilon}}^{t r}=\epsilon_{L} \dot{\xi} \boldsymbol{n}
$$

where $\epsilon_{L}$ is a material constant, which represents the maximum inelastic strain, and $\boldsymbol{n}$ is the normal to the transformation surface, and defined by

$$
\boldsymbol{n}=\sqrt{\frac{3}{2}}\left(\frac{\boldsymbol{s}}{\bar{\sigma}}\right)
$$

where the effective stress is given by

$$
\bar{\sigma}=\sqrt{\frac{3}{2} s: s} .
$$

Similarly, for the martensite to austenite transformation, the loading surfaces are defined as

$$
\begin{aligned}
F_{A}(\boldsymbol{\sigma}, T) & =\sqrt{3 J_{2}}-C_{A} T, \\
F_{A_{s}}(\boldsymbol{\sigma}, T) & =\sqrt{3 J_{2}}-C_{A}\left(T-A_{s}\right), \\
F_{A_{f}}(\boldsymbol{\sigma}, T) & =\sqrt{3 J_{2}}-C_{A}\left(T-A_{f}\right),
\end{aligned}
$$

where $C_{A}$ denotes the slope of the one-dimensional stress-temperature diagram for austenite behavior. As in the forward transformation, the product $F_{A_{s}} F_{A_{f}}$ is negative only for an evolving backward transformation. Evolution of the martensite fraction during the backward transformation is 


$$
\dot{\xi}=-\frac{\left\langle-F_{A_{s}} F_{A_{f}}\right\rangle}{\left|F_{A_{s}} F_{A_{f}}\right|} \frac{\xi}{F_{A_{f}}}\left\langle-\dot{F}_{A}\right\rangle,
$$

and the flow rule is the same as in eq. (5).

Finally, the constitutive law is given by

$$
\boldsymbol{\sigma}=\mathbf{C}(\xi)\left(\boldsymbol{\epsilon}-\boldsymbol{\epsilon}^{t r}\right)
$$

where $\mathbf{C}$ is the standard isotropic stiffness matrix but its components depend upon the internal variable $\xi$ as

$$
E=E_{A}+\xi^{m}\left(E_{M}-E_{A}\right)
$$

and

$$
\nu=\nu_{A}+\xi^{m}\left(\nu_{M}-\nu_{A}\right)
$$

where $E_{A}, E_{M}, \nu_{A}$ and $\nu_{M}$ are the Young's moduli and Poisson's ratios of austenite and martensite phases, respectively, and $m$ is a positive material parameter, which controls the slope of the stress-strain curve.

The stress-strain behavior of the material may be separated into several different branches. It is clear that if $\dot{F}_{M}>0$, a forward transformation occurs, while a backward transformation occurs if $\dot{F}_{A}<0$. Since only one internal variable is considered, $\dot{F}_{M}=\dot{F}_{A}$; hence, only one phase transformation is active at any given time. Consequently, the forward and backward transformations may be decoupled into two separate criteria by defining

$$
h_{M}=\frac{\left\langle-F_{M_{s}} F_{M_{f}}\right\rangle}{\left|F_{M_{s}} F_{M_{f}}\right|}, \quad h_{A}=\frac{\left\langle-F_{A_{s}} F_{A_{f}}\right\rangle}{\left|F_{A_{s}} F_{A_{f}}\right|} .
$$




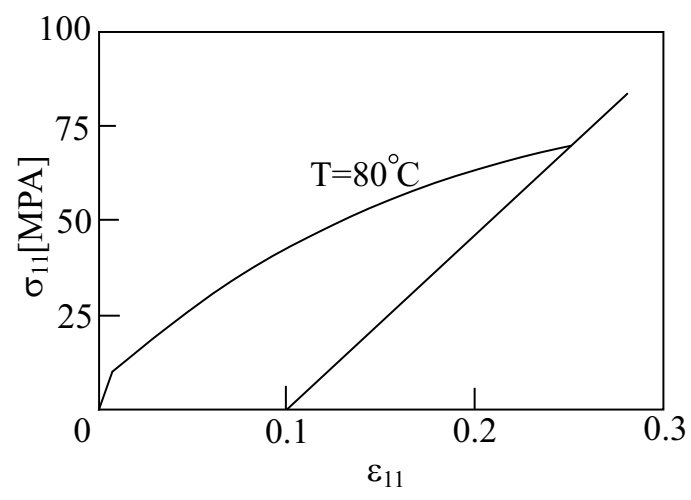

(a)

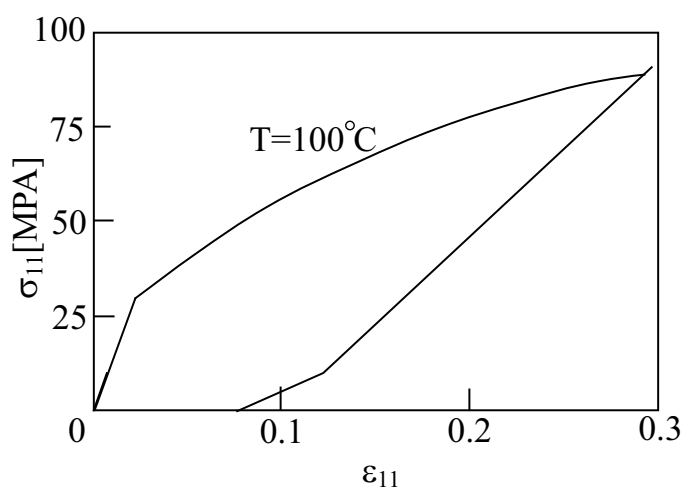

(b)

Fig. 2. One dimensional behavior under monotonic loading for (a) $M_{s}<T<A_{s}$ and (b) $A_{s}<T<A_{f}$.

For a forward transformation,

if $h_{M}=0$, then elastic state

if $h_{M}=1$, then

$$
\begin{array}{lc}
\dot{F}_{M}<0 & \text { elastic unloading } \\
\dot{F}_{M}=0 & \text { neutral loading } \\
\dot{F}_{M}>0 & \text { inelastic loading. }
\end{array}
$$

For a backward transformation,

$$
\begin{array}{cc}
\text { if } h_{A}=0, \text { then } & \text { elastic state } \\
\text { if } h_{A}=1, \text { then } & \\
\dot{F}_{A}>0 & \text { elastic unloading } \\
\dot{F}_{A}=0 & \text { neutral loading } \\
\dot{F}_{A}<0 & \text { inelastic loading. }
\end{array}
$$

A numerical scheme for implementation of the constitutive model is presented in Appendix A.

The response of the material during an isothermal strain-driven uniaxial loadingunloading test is shown in Figs. 2a and 2b for the cases when $M_{s}<T<A_{s}$ and $A_{s}<T<A_{f}$, respectively. In the analyses, the Young's moduli are taken as $E_{M}=500 \mathrm{MPa}$ and $E_{A}=1500 \mathrm{MPa}$, the Poisson's ratios $\nu_{A}=\nu_{M}=0.3$, the transformation temperatures are $M_{s}=70^{\circ} \mathrm{C}, M_{f}=10^{\circ} \mathrm{C}, A_{s}=90^{\circ} \mathrm{C}$, $A_{f}=130^{\circ} \mathrm{C}$, the slopes of the stress-temperature relation $C_{M}=C_{A}=1$ 
$\mathrm{MPa} /{ }^{\circ} \mathrm{C}$, the maximum inelastic strain $\epsilon_{L}=0.1$ and $m=1$ in eqs. (11) and (12). These results agree exactly with those of Panoskaltsis et al. (2004).

\section{Approximate size and shape of the transformation zones}

In this section, the size and shape of the transformation zones are obtained by means of an elastic analysis. This derivation generalizes a similar derivation, presented by Yi and Gao (2000) for plane stress conditions with a different constitutive model. The main assumption is that the material near the crack tip is fully transformed, and this region is sufficiently small, so that the small scale transformation zone assumption may be invoked. Thus, linear elastic fracture mechanics is adequate for this problem. The stress field remote from the crack tip is governed by the applied stress intensity factor $K_{I}^{a p p}$ as

$$
\sigma_{i j}=\frac{K_{I}^{a p p}}{\sqrt{2 \pi r}} f_{i j}(\theta)
$$

where $r$ and $\theta$ are the crack tip polar coordinates, and $f_{i j}$ are given by

$$
\begin{aligned}
f_{x x} & =\cos \frac{\theta}{2}\left(1-\sin \frac{\theta}{2} \sin \frac{3 \theta}{2}\right), \\
f_{y y} & =\cos \frac{\theta}{2}\left(1+\sin \frac{\theta}{2} \sin \frac{3 \theta}{2}\right), \\
f_{x y} & =\sin \frac{\theta}{2} \cos \frac{\theta}{2} \cos \frac{3 \theta}{2},
\end{aligned}
$$

see, for example, Anderson (2005, p. 44). Since the material in the vicinity of the crack tip is fully transformed, there is a disturbance to the stress field in this region. As a result, the stress field is governed by a crack tip stress intensity factor $K_{I}^{\text {tip }}$ with

$$
\sigma_{i j}=\frac{K_{I}^{t i p}}{\sqrt{2 \pi r}} f_{i j}(\theta)
$$

The crack propagates when $K_{I}^{t i p}$ reaches the fracture toughness $K_{I c}$. The toughness change due to the transformation is given by

$$
\Delta K_{I}=K_{I}^{a p p}-K_{I}^{t i p}=K_{I}^{a p p}-K_{I c} .
$$


If $\Delta K>0$, transformation toughening occurs.

By using the $J$-integral, it may be shown that $\Delta K=0$ for the initial transformation zone, where there is no crack growth. Since the $J$-integral is not defined for the case of unloading, it is assumed that $K_{I}^{a p p}$ is monotonically increasing. This is similar to the applicability of the $J$-integral to elasto-plastic cracked bodies which follow a deformation plasticity theory. For plane strain conditions, on a contour $\Gamma$ chosen remote from the crack tip, $J$ is evaluated as

$$
J=\frac{1-\nu^{2}}{E}\left(K_{I}^{a p p}\right)^{2}
$$

while an identical calculation for a contour just outside the transformation zones yields

$$
J=\frac{1-\nu^{2}}{E}\left(K_{I}^{t i p}\right)^{2}
$$

By path independence, it follows that $K_{I}^{t i p}=K_{I}^{a p p}$, or $\Delta K=0$.

Next, the boundary of the martensite transformation zone is determined. It is affected by both the remote stress intensity factor $K_{I}^{a p p}$, and the stress intensity factor governing the region near the crack tip $K_{I}^{t i p}$. The boundary of the transformation zone may be determined as the average value of $K_{I}^{a p p}$ and $K_{I}^{t i p}$ (see Evans, 1984), so that

$$
K_{I}=\frac{K_{I}^{a p p}+K_{I}^{t i p}}{2}
$$

For mode I loading, using the assumption of a small scale transformation zone, the stress field is given by

$$
\begin{aligned}
\sigma_{x x} & =\frac{K_{I}}{\sqrt{2 \pi r}} f_{x x}(\theta), \\
\sigma_{y y} & =\frac{K_{I}}{\sqrt{2 \pi r}} f_{y y}(\theta), \\
\sigma_{x y} & =\frac{K_{I}}{\sqrt{2 \pi r}} f_{x y}(\theta),
\end{aligned}
$$


with

$$
\sigma_{z z}=\left\{\begin{array}{cc}
0, & \text { plane stress } \\
\nu\left(\sigma_{x x}+\sigma_{y y}\right), & \text { plane strain }
\end{array}\right.
$$

and the functions $f_{i j}$ are given in eq. (17).

The limit function of Panoskaltsis et al. (2004) is given in eq. (3) for a forward transformation. Substituting eqs. (23) and (24) into eq. (3) ${ }_{2}$, with some manipulations, yields

$$
r_{f}=\frac{1}{2 \pi}\left[\frac{K_{I}}{C_{M}\left(T-M_{f}\right)}\right]^{2} R(\theta),
$$

where

$$
R(\theta)=\cos ^{2} \frac{\theta}{2}\left[\kappa^{*}+3 \sin ^{2} \frac{\theta}{2}\right]
$$

and

$$
\kappa^{*}=\left\{\begin{array}{cc}
1, & \text { plane stress } \\
(1-2 \nu)^{2}, & \text { plane strain }
\end{array}\right.
$$

A similar derivation was carried out by Yi and Gao (2000) for a martensite transformation under plane stress conditions. Equation (25) describes the transformation zone closest to the crack tip for $\xi=1$ which is a full martensite transformation (see Fig. 3a for plane stress conditions). In Fig. 3, the length parameters have been normalized with respect to the quantity

$$
L=\frac{1}{2 \pi}\left[\frac{K_{I}}{C_{M}\left(T-M_{f}\right)}\right]^{2}
$$

so that $\hat{x}=x / L, \hat{y}=y / L$, and $R(\theta)=r_{f} / L$. As expected, the same zone is found for plastically deforming material with $C_{M}\left(T-M_{f}\right)$ replaced by the yield stress $\sigma_{Y}$. 


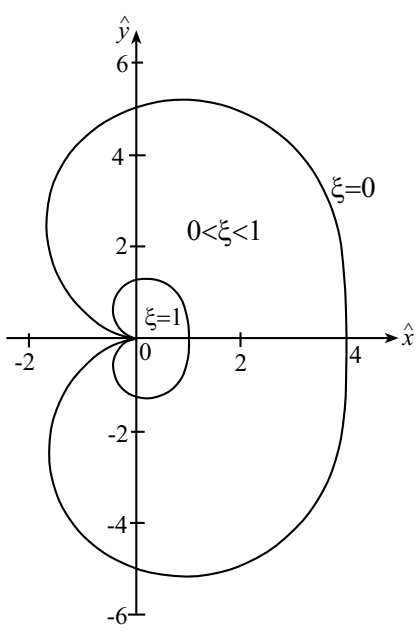

(a)

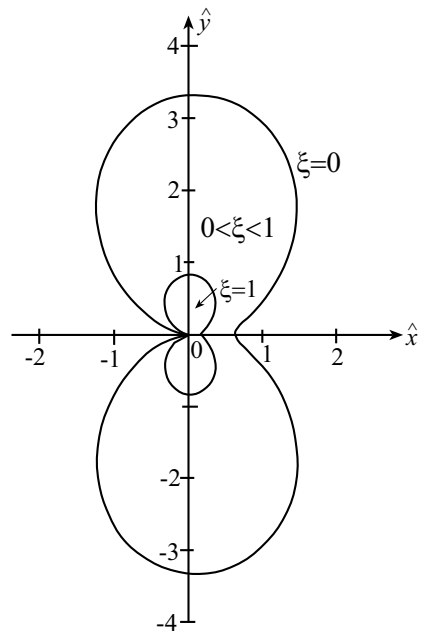

(b)

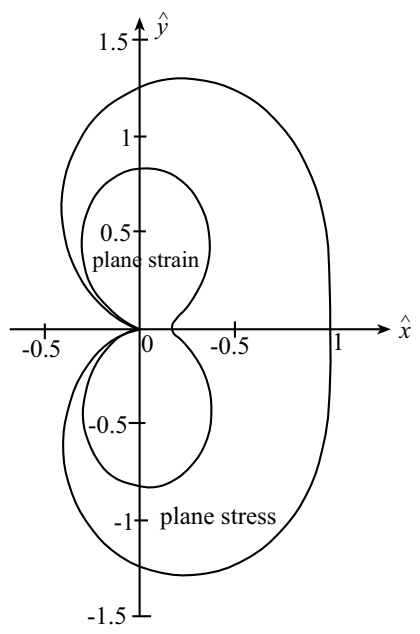

(c)

Fig. 3. The shape of the initial transformation zone for (a) plane stress conditions with $T>A_{f}$ or $A_{f}>T>A_{s}$ or $A_{s}>T>M_{s}(\rho>1)$, (b) plane strain conditions with $\nu=0.3$ and $T>A_{f}$ or $A_{f}>T>A_{s}$ or $A_{s}>T>M_{s}(\rho>1)$, and (c) plane stress and strain conditions with $\nu=0.3$ and $M_{f}<T<M_{s}(\rho<-1)$.

As an example, consider a temperature such that $T>A_{f}$, as shown in Fig. 1b. The curve described by eq. (25) corresponds to the locus of points at which the effective stress is at point 3 in this figure. For the value of the effective stress at point 2 in Fig. 1b, the curve describing the transformation zone is given by

$$
r_{s}=\frac{1}{2 \pi}\left[\frac{K_{I}}{C_{M}\left(T-M_{s}\right)}\right]^{2} R(\theta) .
$$

This situation corresponds to $\xi=0$ in which the material outside of this zone is completely austenitic (see Fig. 3a). The material between these two curves is a mixture of martensite and austenite with $0<\xi<1$. To represent the curve described by eq. (29), the normalized parameter

$$
\rho=\frac{T-M_{f}}{T-M_{s}}
$$

is defined. Thus,

$$
r_{s}=\rho^{2} r_{f}
$$

It may be noted that the curves in Fig. 3a are valid for $\rho>1$. The value of $\rho=2$ was used to obtain the graph, hence the values shown on the axes. The 
same curves may be obtained in Fig. 3a for $A_{f}>T>A_{s}$ or $A_{s}>T>M_{s}$. For $T>A_{f}$ or $A_{f}>T>A_{s}$ or $A_{s}>T>M_{s}$, the curves are redrawn for plane strain conditions with $\rho=2$ and Poisson's ratio $\nu=0.3$ in Fig. 3b. It may be observed that the scales are different for plane stress and plane strain. As expected, the transformation zones governed by plane strain are smaller than those governed by plane stress. For $M_{f}<T<M_{s}$, there is only a fully transformed region near the crack tip. The material outside the transformation zone is a mixture of austenite and martensite $(0<\xi<1)$. For this case, a comparison of the transformation zones for plane stress and plane strain is illustrated in Fig. 3c $(\rho=-2, \nu=0.3)$. Finally, for $T<M_{f}$, there is no phase transformation between austenite and martensite, and reorientation of martensite variants occurs. The chosen model in this study does not describe this phenomenon.

It may be noted that in this section the shape and size of the transformation zones are derived with the aid of linear elastic fracture mechanics. However, in the sequel, the behavior of the transformation regions is governed by a cohesive zone model. The shape and size of the initial transformation zones are similar to those in Fig. 3b. The above derivation may be considered as an approximation of the numerical results.

\section{A cohesive interface element}

To obtain more exact transformation zones, as well as the transformation toughening behavior of SMAs, a cohesive zone model is employed to simulate quasi-static crack propagation. This model assumes the existence of a zone ahead of the crack tip where stresses are non-zero but not infinite, and relative displacements (i.e. the crack opening or sliding displacement) can occur. This method may be numerically implemented using an interface element, with different traction-separation laws.

There are several cohesive zone models that are suitable for mixed mode deformations. In general, laws are written for each mode and then coupled for mixed modes, either by using an effective displacement or by defining coupling parameters. For example, the exponential cohesive law of Xu and Needleman (1993) is governed by two coupling parameters, which relate the ratio between the energy release rates of pure modes I and II and the relative displacements. However, it was recently shown by van den Bosch et al. (2006) that this model does not satisfy zero traction of both modes simultaneously at crack propagation, unless the energy release rates of each are chosen equal. Unfortunately, this assumption is not generally correct. On the other hand, a non-dimensional effective crack separation was assumed by Tvergaard and Hutchinson (1993) which overcomes this problem. 


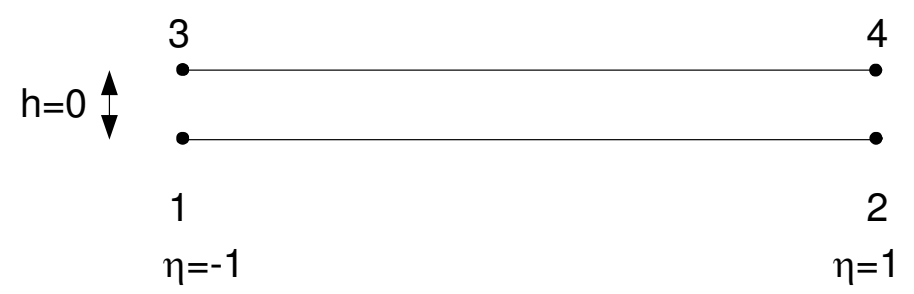

Fig. 4. A four noded linear interface element.

In this study, the bilinear law which corresponds to an appropriate failure criterion, as exploited by Alfano and Crisfield (2001) is employed. This element is one-dimensional, which is suitable for two-dimensional analyses, and consists of a fracture criterion for mixed mode delamination. The interface element is considered to be of zero thickness $(h=0)$ in the non-deformed state and is modeled by 4-noded linear elements, as shown in Fig. 4. At a given point of the interface, the relative displacement and the corresponding traction are denoted by $\boldsymbol{\delta}=\left[\delta_{1}, \delta_{2}\right]^{T}$ and $\mathbf{t}=\left[t_{1}, t_{2}\right]^{T}$, respectively. The relative displacement vector is obtained from

$$
\boldsymbol{\delta}(\eta)=\mathbf{B}(\eta) \mathbf{p}
$$

where $\mathbf{p}=\left[\mathbf{p}_{1}^{T}, \mathbf{p}_{2}^{T}, \mathbf{p}_{3}^{T}, \mathbf{p}_{4}^{T}\right]^{T}, \mathbf{p}_{j}=\left[u_{j}, v_{j}\right]^{T}, j$ refers to the global reference system and $u_{j}$ and $v_{j}$ are the displacements in the $x$ and $y$-directions, respectively. The matrix $\mathbf{B}$ is given as

$$
\mathbf{B}=\left(\begin{array}{cccccccc}
-N_{1} & 0 & -N_{2} & 0 & N_{1} & 0 & N_{2} & 0 \\
0 & -N_{1} & 0 & -N_{2} & 0 & N_{1} & 0 & N_{2}
\end{array}\right),
$$

where $N_{1}=(1-\eta) / 2$ and $N_{2}=(1+\eta) / 2$ are the linear one-dimensional shape functions.

The first step in the derivation of the interface element is to describe the uncoupled traction-separation laws for modes I and II. The laws, illustrated in Fig. 5, are bilinear. Since in mode I, penetration must be avoided, it is not symmetric. The first part of the constitutive law is a linear relation between the traction and the relative displacement with a slope $K_{i}$ (where $i=1,2$ for modes I and II, respectively). Hence, in this region,

$$
t_{i}=K_{i} \delta_{i}, \quad \delta_{i} \leq \delta_{0 i}
$$

no summation implied. The parameter $\delta_{0 i}$ is the elastic limit of the relative displacement, so that the maximum traction is given by $t_{0 i}=K_{i} \delta_{0 i}$. The second part of the constitutive law exhibits softening behavior. As $\delta_{i}$ approaches 


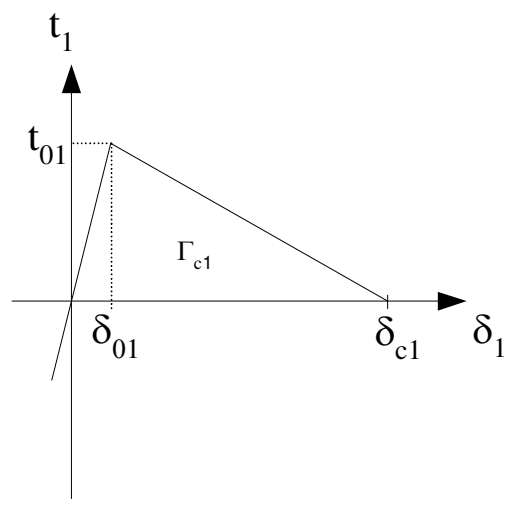

(a)

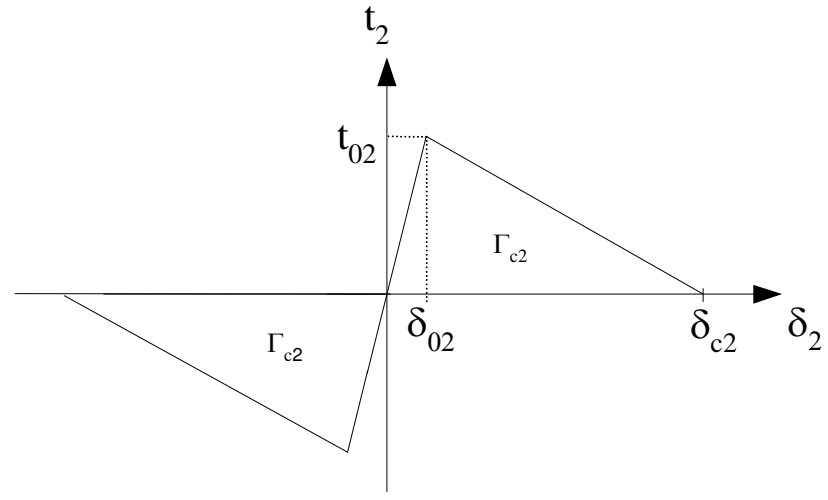

(b)

Fig. 5. Traction-separation constitutive law for (a) mode I and (b) mode II deformation.

$\delta_{c i}$, its critical value, the corresponding traction vanishes; hence, the crack propagates a small increment. Concurrently, the critical energy release rate or Griffith's energy $\Gamma_{c i}$ is reached.

Alfano and Crisfield (2001) claim that, below sufficiently small values, $\delta_{0 i}$ has a very small effect on overall material response. The choice of this parameter is related to the stiffness parameters $K_{i}$ and the cohesive strengths $t_{0 i}$. However, Alfano and Crisfield (2001) suggest choosing $K_{i}$ as penalty parameters, so that $\delta_{0 i}$ may be taken as small as possible, as long as it is within a range that avoids ill-conditioning of the tangent modulus matrix. This has been checked here and seen to be correct. Hence, $\delta_{0 i}$ may be chosen arbitrarily, as long as $\delta_{0 i} \ll \delta_{c i}$. In this case, the energy release rate results in

$$
\Gamma_{c i}=\frac{1}{2} t_{0 i} \delta_{c i}
$$

The remainder of the constitutive law may be expressed by

$$
t_{i}=K_{i} \frac{\delta_{0 i}}{\delta_{0 i}-\delta_{c i}}\left(\delta_{i}-\delta_{c i}\right), \quad \delta_{0 i}<\delta_{i}<\delta_{c i}
$$

and

$$
t_{i}=0, \quad \delta_{i} \geq \delta_{c i}
$$

for complete debonding.

In Alfano and Crisfield (2004), mixed mode deformation was treated by two 
constitutive laws which were related to each other via a failure criterion. The fracture criterion was taken as

$$
\left(\frac{\Gamma_{1}}{\Gamma_{c 1}}\right)^{\alpha / 2}+\left(\frac{\Gamma_{2}}{\Gamma_{c 2}}\right)^{\alpha / 2}=1
$$

where $\Gamma_{1}$ and $\Gamma_{2}$ are the cohesive energies in modes I and II, respectively, $\Gamma_{c 1}$ and $\Gamma_{c 2}$ are the critical cohesive energies of the uncoupled constitutive laws (see Fig. 5), that are determined from appropriate tests for each mode and $2 \leq \alpha \leq 4$. Details are presented in Appendix B.

To implement the interface element, the internal force vector $\mathbf{q}$ is given by

$$
\mathbf{q}=\int_{A} \mathbf{B}^{T} \mathbf{t} d A=\int_{-1}^{1} \mathbf{B}^{T} \mathbf{t} \ell d \eta
$$

where $\ell$ is the length of the interface element in the global reference system. The finite element equation is given in incremental form as

$$
\Delta \mathbf{q}=\mathbf{D}_{t} \Delta \mathbf{p}
$$

where

$$
\mathbf{D}_{t}=\int_{A} \mathbf{B}^{T} \mathbf{K}_{t} \mathbf{B} d A=\int_{-1}^{1} \mathbf{B}^{T} \mathbf{K}_{t} \mathbf{B} \ell d \eta
$$

and $\mathbf{K}_{t}$ is given in eq. (B.7).

A difficulty with this coupled law is that for complete debonding it is necessary that $\mathbf{t}=0$ which is usually not obtained simultaneously for modes I and II. However, choosing $t_{1}=0$ with $t_{2}=0$ implies that $F_{11}=F_{22}$ (see Appendix B). Since $\delta_{01}$ and $\delta_{02}$ can be chosen arbitrarily in such a way that ill-conditioning is avoided, then assuming $\delta_{01} / \delta_{c 1}=\delta_{02} / \delta_{c 2}$ leads to $t_{1}=t_{2}=0$.

From a computational point of view, Schellekens and de Borst (1993) showed that a Gauss quadrature integration rule is not recommended for interface elements. Instead, a Newton-Cotes integration rule was found to exhibit superior performance. In particular, for linear elements, it was shown that when a Gauss quadrature integration rule was used, there was coupling between the 


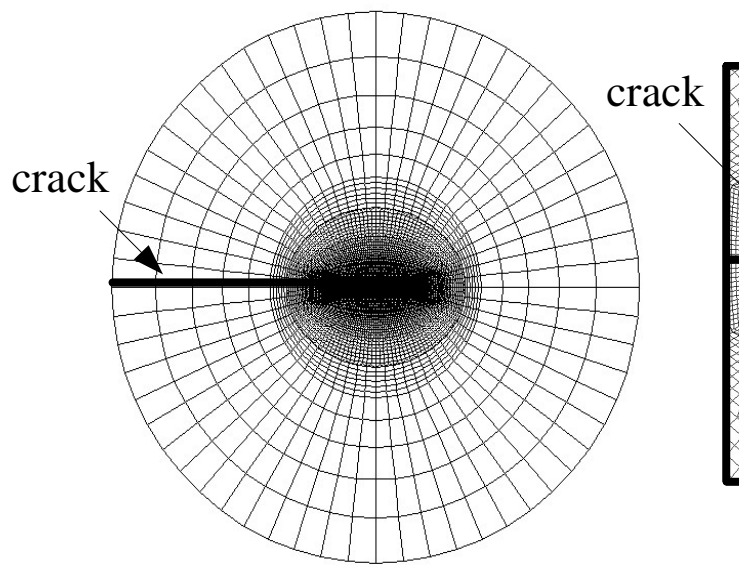

(a)

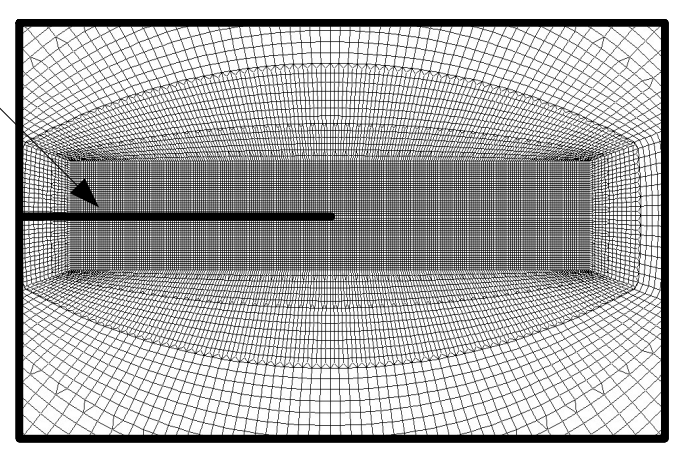

(b)

Fig. 6. (a) The finite element mesh used in the analyses with 21,260 4-noded isoparametric elements and 124 linear 4-noded interface elements. (b) The crack tip region.

degrees of freedom of nodes in opposing sides of the interface element which results in an oscillation of the traction profile. This phenomenon however, does not occur when using a Newton-Cotes integration rule. Furthermore, Alfano and Crisfield (2001) showed that a high number of integration points does not necessarily yield a more accurate estimation of the stiffness and traction. They found that in some cases, the increased number of integration points turns out to be less robust; consequently, a smaller incremental time step is needed. Conversely, higher stability is achieved with two integration points and a larger average time step.

\section{Problem formulation and numerical results}

In this section, the relations between the cohesive zone fracture parameters and the crack growth resistance of shape memory alloys are determined by means of resistance curves for two temperature ranges. To this end, plane strain, mode I analyses are carried out for conditions of small scale transformation. The body considered is a disk of radius and crack length $R_{0}$ as shown in Fig. 6a. Both constitutive equations of shape memory alloys and the cohesive zone interface elements (described in Sections 2 and 4) are implemented as user supplied subroutines in ADINA (Bathe, 2006). The finite element mesh consists of 21,620 4-noded isoparametric elements with 21,671 nodal points, as well as 124 linear 4-noded interface elements with 250 nodal points (see Figs. 6a and 6b). A two point Newton-Cotes integration rule is used for the interface elements, together with the failure criterion in eq. (38) for $\alpha=2$, namely

$$
\Gamma_{1}=\Gamma_{c 1}
$$


The interface elements are located along the path of the propagating crack (on the symmetry line). The bilinear traction-separation law, described in Appendix B, is implemented with these interface elements. Since only mode I deformation is considered, $\boldsymbol{\delta}=\left[\delta_{1}, 0\right]^{T}$, the traction-separation law in eq. (B.3) reduces to

$$
t_{1}(\tau)=\left\{\begin{array}{cc}
K_{11} \delta_{1}(\tau) & \bar{\gamma}(\tau) \leq 0 \\
{\left[1-D_{11}(\tau) C_{11}(\tau)\right] K_{11} \delta_{1}(\tau)} & \bar{\gamma}(\tau)>0
\end{array}\right.
$$

with $t_{2}(\tau)=0$ and

$$
\gamma\left(\tau^{\prime}\right)=\frac{<\delta_{1}\left(\tau^{\prime}\right)>}{\delta_{01}}-1=\frac{<\delta_{1}\left(\tau^{\prime}\right)>-\delta_{01}}{\delta_{01}}
$$

While displacement boundary conditions are applied to the outer boundary of the body, the crack faces remain traction free. The displacements are taken as the first term of the asymptotic displacement field with a prescribed value for the stress intensity factor, and material properties of the austenite, which is the stable phase in the far field. The far field stress intensity factor $K_{I}^{a p p}$ is monotonically increased until crack propagation is reached based upon eq. (42). Upon this initial crack propagation, the applied stress intensity factor $K_{I}^{a p p}$ is set to $K_{I}^{\text {tip }}$ and used as a reference; the reader may recall that $K_{I}^{a p p}=K_{I}^{\text {tip }}$ for a stationary crack (see Section 3 for further details). For each increment of crack propagation, the analysis is stopped and a new displacement field is prescribed, with respect to the new location of the crack tip and to the new value of $K_{I}^{a p p}$.

An important issue that is worth discussion is selection of the crack tip position. Unlike traditional fracture mechanics, where the crack tip is defined by a single point, a cohesive zone represents a region of separation; hence, the location of the crack tip should be somewhere within this region. For a bilinear traction separation law, as shown in Fig. 5a, one may consider three different locations of the crack tip, namely $\delta_{t i p}=0, \delta_{t i p}=\delta_{0}$ or $\delta_{t i p}=\delta_{c}$, as discussed in Li and Chandra (2003) for crack tip plasticity. Note that $\delta \equiv \delta_{1}$ and $t \equiv t_{1}$. In their study, they considered $\delta_{t i p}=\delta_{0}$ as the location of the crack tip. In particular, they claimed that the process zone ahead of the crack tip is associated with the cohesive energy of the region $0 \leq \delta \leq \delta_{0}$, while the wake of the crack is associated with the energy between $\delta_{0}$ and $\delta_{c}$. Other choices, such as $\delta_{\text {tip }}=0$ or $\delta_{\text {tip }}=\delta_{c}$, cannot properly describe the different macroscopic processes that occur in the region of the crack tip. For instance, choosing $\delta_{\text {tip }}=0$ as the location of the crack tip suggests that the entire energy is absorbed in 
Table 1

Material properties that were used for the material model (see Section 2).

\begin{tabular}{llllllllll}
\hline property & $E_{A}=E_{M}$ & $\nu_{A}=\nu_{M}$ & $m$ & $C_{A}=C_{M}$ & $A_{s}$ & $A_{f}$ & $M_{s}$ & $M_{f}$ & $\Gamma_{c}$ \\
\hline value & $20.2 \mathrm{GPa}$ & 0.3 & 1 & $3 \mathrm{MPa} /{ }^{\circ} \mathrm{C}$ & $10^{\circ} \mathrm{C}$ & $15^{\circ} \mathrm{C}$ & $-1^{\circ} \mathrm{C}$ & $-5^{\circ} \mathrm{C}$ & $1 \mathrm{kN} / \mathrm{m}$ \\
\hline
\end{tabular}

the wake of the propagating crack. However, there are macroscopic processes such as damage, that occur ahead of the crack tip. On the other hand, choosing $\delta_{t i p}=\delta_{c}$ as the crack tip implies that all of the cohesive energy is used for separation, and there is no active wake behind the crack tip. This is also less plausible, since it has been shown by Ritchie (1999) that micromechanical processes are active in the wake, as well as ahead of the crack. Consequently, the chosen crack tip position in this study is the point $\delta_{t i p}=\delta_{0}$ corresponding to the maximum traction $t_{0}$.

Next, another reference length is defined as

$$
L_{c}=\frac{\left(1-2 \nu^{*}\right)^{2}}{2 \pi}\left[\frac{K_{I c}}{C_{M}\left(T-M_{s}\right)}\right]^{2}
$$

which denotes the length of the outer transformation zone along the $x$-axis between the austenitic and partially transformed material for plane strain conditions (see Fig. 3b). In eq. (45), $\nu^{*}=\left(\nu_{A}+\nu_{M}\right) / 2$, where $\nu_{A}$ and $\nu_{M}$ are the Poisson's ratios of the austenite and martensite phases, respectively. Moreover, the length $\Delta_{0}$ is defined as the size of an element in the vicinity of the crack tip. In all calculations, a resolution of at least three elements in the transformation zones is attained. This resolution was examined and found to result in convergence. To ensure a small scale transformation, the ratio $R_{0} / \Delta_{0}=625$ is chosen; recall that $R_{0}$ is the radius of the body. The material properties used in this part of the study are presented in Table 1.

The normalized intensity of the transformation $\omega$ is defined by

$$
\omega=\frac{4 E^{*} \epsilon_{L}^{2}}{9 C_{M}\left(T-M_{s}\right)}\left(\frac{1+\nu^{*}}{1-\nu^{*}}\right)
$$

where $E^{*}=\left(E_{A}+E_{M}\right) / 2, E_{A}$ and $E_{M}$ are the Young's moduli of the austenite and martensite phases, respectively, and $\epsilon_{L}$ represents the maximum transformation strain as given in eq. (5). In this study, the values of $\omega$ are taken as 5 and 20 . 


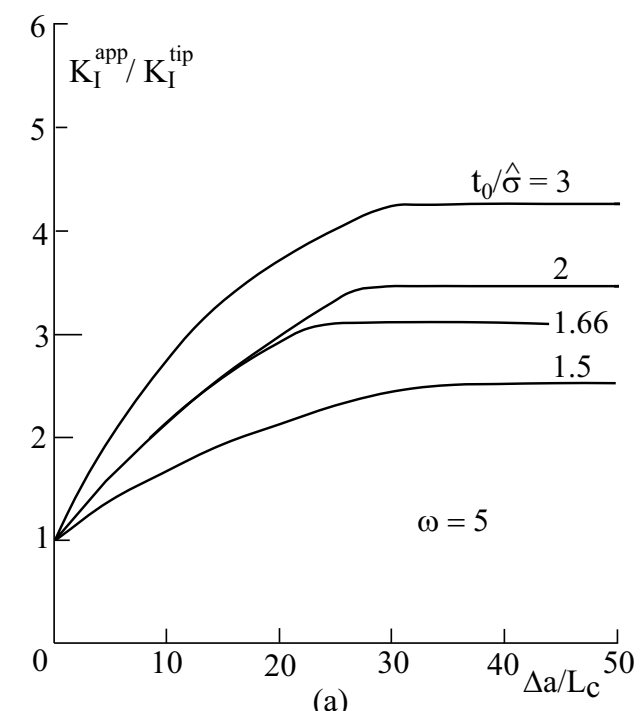

(a)

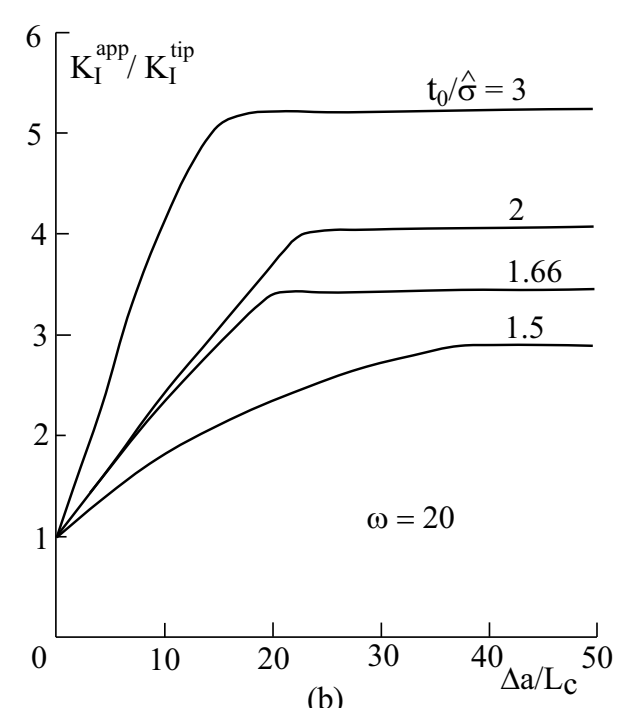

(b)

Fig. 7. Crack growth resistance curves for $M_{s}<T<A_{s}$ and different values of the normalized maximum separation traction $t_{0} / \hat{\sigma}$ with (a) $\omega=5$ and (b) $\omega=20$.

In Figs. 7a and 7b, the crack resistance curves (R-curves) for these values of $\omega$ are illustrated for a constant temperature of $M_{s}<T<A_{s}$; hence, no reverse transformation occurs. A reference temperature $T=0^{\circ} \mathrm{C}$ is chosen. It may be noted that for this temperature and the temperature values in Table $1, \rho=5$ in eq. (30). Although the outer transformation zone in Fig. 3 is still small with respect to $R_{0}$, the proportions between the inner and outer transformation zones have changed. Recall that $\rho=2$ was used to obtain the curves in Fig. 3. Maximum transformation strains $\epsilon_{L}$ of 0.03 and 0.06 are used for $\omega=5$ and 20 , respectively. In both figures, R-curves are obtained for different values of $t_{o} / \hat{\sigma}$, where $t_{0}$ is the maximum normal traction for mode I separation (see Fig. 5a), and

$$
\hat{\sigma}=C_{M}\left(T-M_{s}\right)
$$

is the effective stress that induces the isothermal martensitic transformation. In all cases, transformation toughening is observed. After the crack has advanced at least one order of magnitude past the original transformation zones as seen in Fig. $7 \mathrm{a}, K_{I}^{a p p}$ reaches a steady state value, denoted here by $K_{s s}$. However, it is pointed out that with the analyses presented here, transformation softening cannot occur after a peak value of $K_{I}^{a p p} / K_{I}^{t i p}$ is reached. With the interface elements used in the analyses, once the R-curve reaches its highest value, the crack propagates rapidly. Nevertheless, from an engineering point of view, $K_{s s}$ is an appropriate design parameter.

Values of $K_{s s} / K_{I}^{t i p}$ are presented in Table 2 for various values of $t_{0} / \hat{\sigma}$. It is clear that $K_{s s} / K_{I}^{t i p}$ increases with increasing $t_{0} / \hat{\sigma}$. For higher values of $t_{0} / \hat{\sigma}$, a larger transformation zone develops ahead of the crack tip, and higher 
Table 2

Normalized steady state stress intensity factors $K_{s s} / K_{I}^{\text {tip }}$ as a function of the maximum tensile stress ratio $t_{0} / \hat{\sigma}$ in the cohesive zone for two values of the normalized maximum transformation strain $\omega$ in different temperatures ranges.

\begin{tabular}{r|r|rrrr}
\hline & \multicolumn{1}{|c|}{$t_{0} / \hat{\sigma}$} & 1.5 & 1.66 & 2.0 & 3.0 \\
\hline \multirow{2}{*}{$M_{s}<T<A_{s}$} & $\omega=5$ & $K_{s s} / K_{I}^{t i p}=2.48$ & 3.07 & 3.43 & 4.22 \\
& 20 & 2.84 & 3.40 & 4.01 & 5.18 \\
\hline$A_{s}<T<A_{f}$ & $\omega=5$ & 2.03 & 2.35 & 2.45 & 2.64 \\
& 20 & 2.52 & 2.97 & 3.19 & 3.30 \\
\hline
\end{tabular}

energy dissipation occurs upon crack propagation. As a result, the steady state resistance $K_{s s}$ increases. For $t_{0} / \hat{\sigma}$ higher than the values studied here, the transformation zone is no longer small, and crack tip blunting should be considered. That is, with increasing values of $K_{I}^{a p p}$, the crack undergoes increased blunting, and its propagation becomes slower in the sense that more applied load is needed for an increment of crack propagation. For $t_{0} / \hat{\sigma}=1.5$, for example, a small transformation zone develops and the energy dissipation is small compared to that obtained when $t_{0} / \hat{\sigma}=3$. For values of $t_{0} / \hat{\sigma}<1.5$, the $R$-curve is nearly flat.

Similar behavior is observed for $\omega=20$, as presented in Table 2 and Fig. 7b. The reader may note that the values of $K_{s s} / K_{I}^{t i p}$ obtained here are higher with respect to those for $\omega=5$. It may be observed in eq. (46) that the intensity parameter $\omega$ is strongly dependent upon the transformation strain $\epsilon_{L}$. Hence, higher values of $\omega$ are associated with larger transformation strains and the energy dissipation resulting from the transformation zone is significantly higher. Consequently, the values of $K_{s s} / K_{I}^{t i p}$ increase with the ratio $t_{0} / \hat{\sigma}$.

The effect of a reverse transformation on the resistance curves is examined in Figs. 8a and 8b for $\omega=5$ and $\omega=20$, respectively. An isothermal transformation with $A_{s}<T<A_{f}$ (see Fig. 1b) is examined in this case. A nondimensional parameter

$$
\beta=\frac{T-A_{s}}{A_{f}-A_{s}}
$$

is introduced. This parameter measures the amount of reverse transformation, so that for $\beta \leq 0$, no reverse transformation occurs, and $\beta=1$ yields full recovery of the transformation strains. In the analyses that are illustrated in Figs. $8 \mathrm{a}$ and $8 \mathrm{~b}, \beta=0.5$ ( or $T=12.5^{\circ} \mathrm{C}$ ). For this temperature value, the parameter $\rho$ in eq. (30) is 1.3. Hence, the inner and outer transformation zones are closer to each other as compared with those in the previous example. 


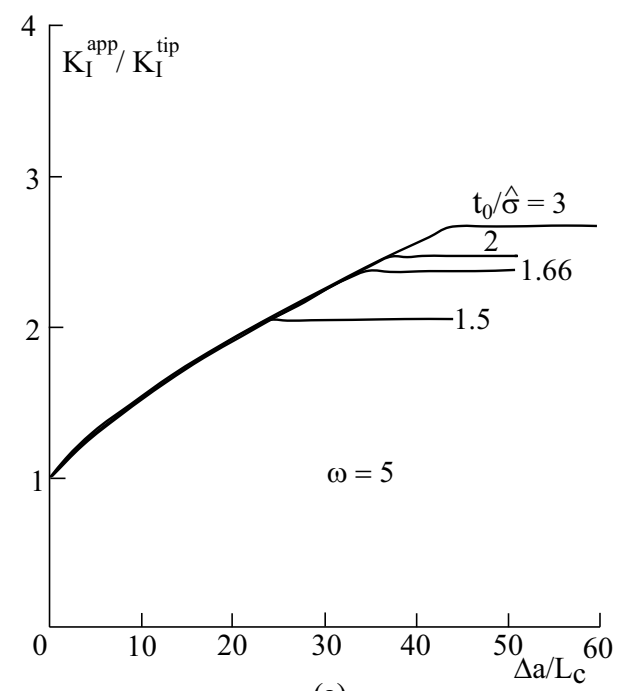

(a)

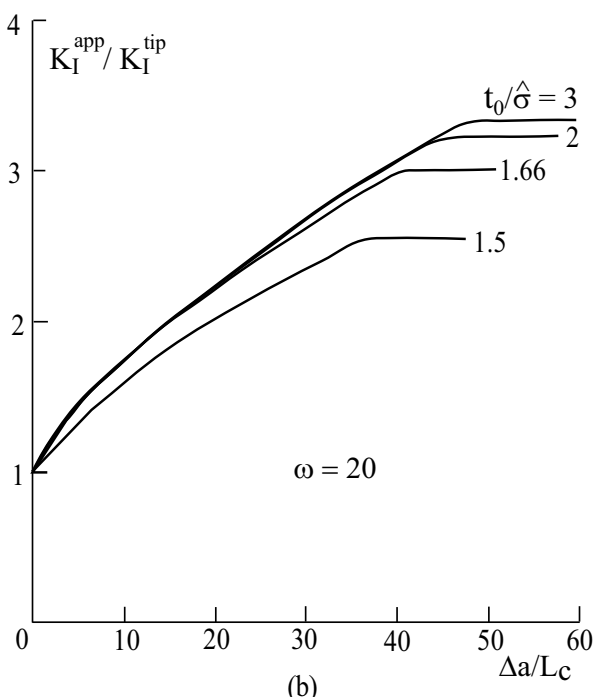

(b)

Fig. 8. Crack growth resistance curves for $A_{s}<T<A_{f}$ and different values of the normalized maximum separation traction $t_{0} / \hat{\sigma}$ with (a) $\omega=5$ and (b) $\omega=20$. A reverse transformation occurs in the wake $(\beta=0.5)$.

The reader should recall that $L_{c}$ is only an approximation of the length of the transformation zone (in the presence of a singularity). However, in the cohesive zone model, the stresses are bounded in the vicinity of the crack tip, and consequently, the stress singularity vanishes. Hence, the actual size of the transformation zones differ in these analyses from the theoretical sizes that are presented in Section 3. Nonetheless, there are at least three finite elements ahead of the crack tip in the transformation zones.

Prior to crack growth, no unloading occurs in the material. The reverse transformation occurs only in the wake of the propagating crack. From Figs. 8a and 8b, as well as Table 2, it is observed that the reverse transformation reduces values of $K_{s s}$ as compared to those obtained in Fig. 7 for a forward transformation only. Note that the scales in Figs. 7 and 8 differ. The energy dissipation in the wake decreases as a result of the reverse transformation. For higher values of $t_{0}$, the reverse transformation is more intensive; hence, less energy dissipation occurs. Since there is still a forward transformation ahead of the crack tip, the values of $K_{s s}$ increase with increasing $t_{0} / \hat{\sigma}$. In Fig. 9a, the initial transformation zones are illustrated, as obtained by the finite element analysis for $\omega=20, t_{0} / \hat{\sigma}=2$ and $\beta=0.5$. Upon crack propagation, a partial recovery of the transformation strains in the wake occurs, as shown in Fig. 9b for these values of the parameters.

Comparison between the results from Figs. 7 and 8 are summarized in Fig. 10. The ratio $K_{s s} / K_{I}^{t i p}$ is plotted vs $t_{0} / \hat{\sigma}$ for different temperature ranges and different values of $\omega$. It may be observed in all cases that $K_{s s} / K_{I}^{t i p}$ increases monotonically with $t_{0} / \hat{\sigma}$. For fixed $\Gamma_{c}, t_{0}$ and $\delta_{0}$, increasing the temperature 


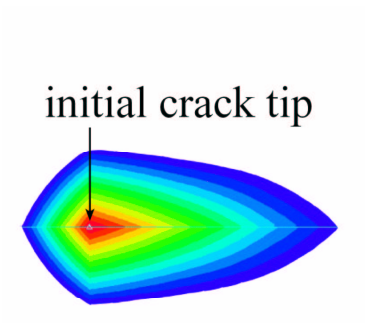

(a)

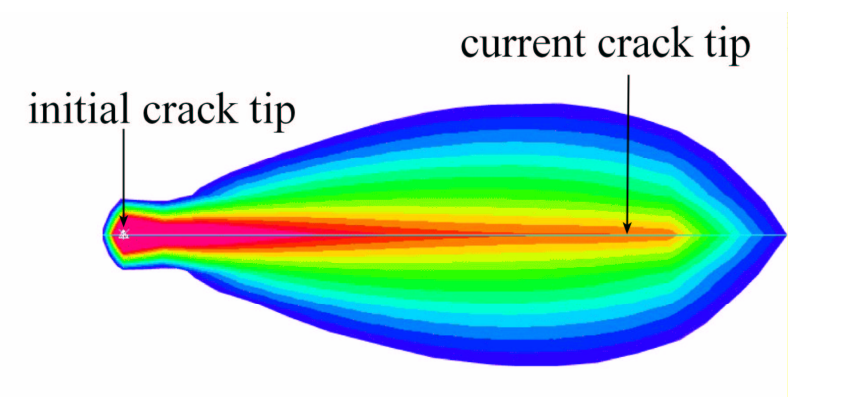

(b)

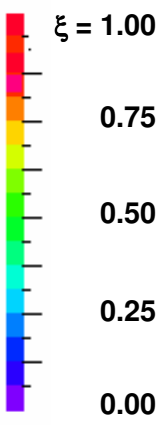

0.00

Fig. 9. For $\omega=20, t_{0} / \hat{\sigma}=2$ and $\beta=0.5$ (a) the initial transformation zone in the vicinity of the crack tip and (b) the transformation zone of a propagating crack. Recovery occurs in the wake.

above $A_{s}$ decreases $K_{s s} / K_{I}^{\text {tip }}$, as well as its slope.

Finally, the effect of the Young's moduli ratio $E_{A} / E_{M}$ is considered. It should be mentioned that this ratio varies for different compositions of shape memory alloys. For example, $E_{A} / E_{M} \approx 1$ in copper based shape memory alloys, while the same ratio for Nitinol is almost 3. The effect of this ratio on the steady state values for different choices of $t_{0} / \hat{\sigma}$ and $\omega=5$ is illustrated in Fig. 11. It may be observed that the ratio $K_{s s} / K_{s s}^{*}$ increases with increase of the moduli mismatch. The parameter $K_{s s}^{*}$ is the steady state stress intensity factor for the case of no material mismatch, i.e. $E_{A} / E_{M}=1$. For $E_{A} / E_{M}=3$, $K_{s s} / K_{s s}^{*}=1.13$ and 1.19 for $t_{0} / \hat{\sigma}=2$ and $t_{0} / \hat{\sigma}=3$, respectively. This behavior conforms with that obtained by Yi and Gao (2000) by means of Eshelby's equivalent inclusion method, together with weight functions.

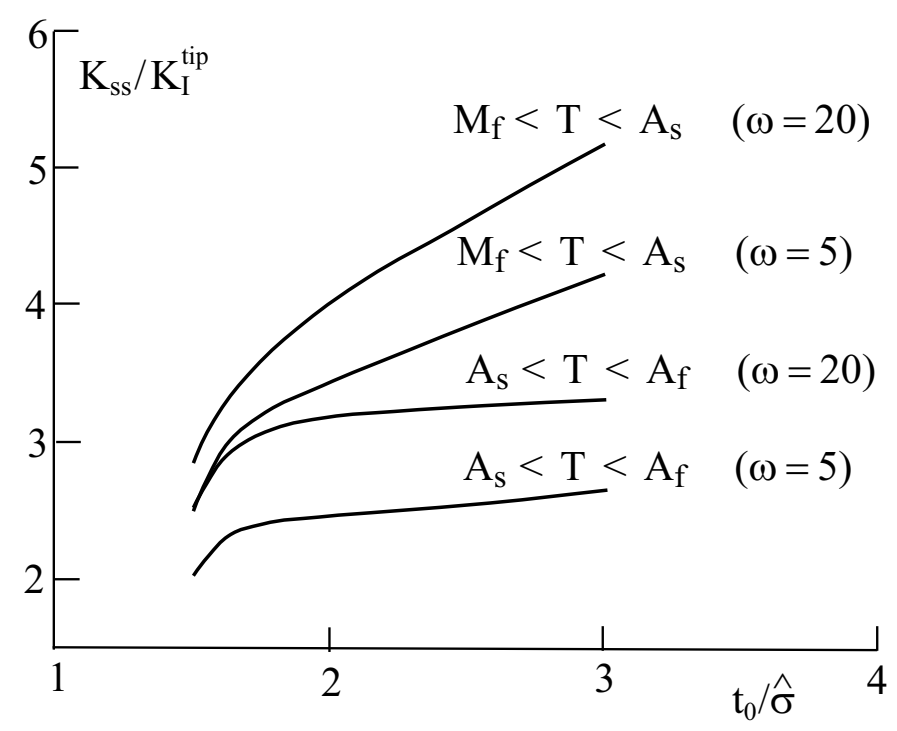

Fig. 10. Steady state transformation toughness as a function of $t_{0} / \hat{\sigma}$ for $\omega=5$ and $\omega=20$ in different temperature ranges. 


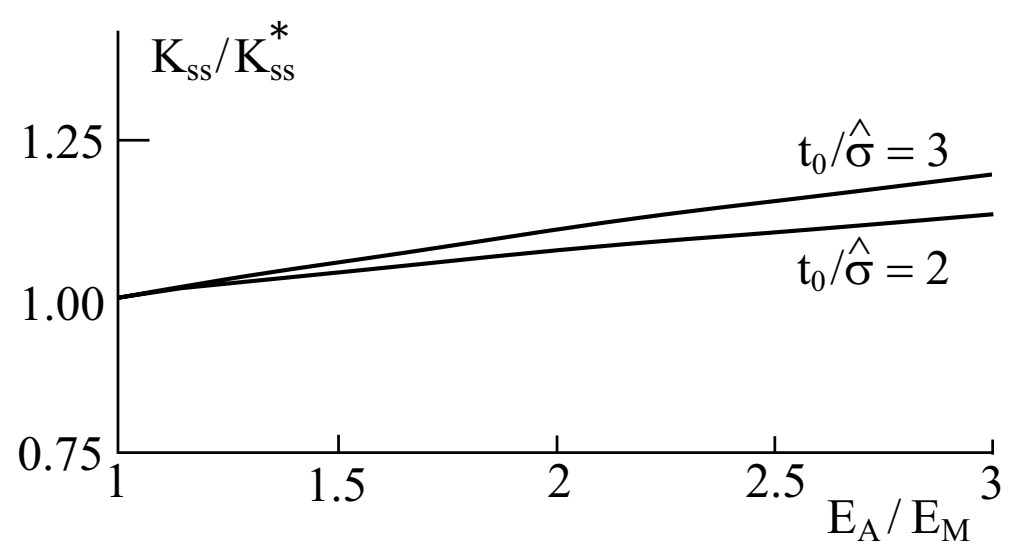

Fig. 11. Steady state transformation toughness as a function of $E_{A} / E_{M}$ for different $t_{0} / \hat{\sigma}$ ratios and $\omega=5$.

\section{Summary and conclusions}

In this investigation, the behavior of cracks in shape memory alloys was studied. Upon isothermal loading, two transformation zones are generally observed in the vicinity of the crack tip: the fully and partially transformed martensite zones. At a distance from the crack tip, there is an austenite region. The zones near the crack tip were derived from the first term of the asymptotic solution for linear elastic material. With this assumption, it was found that the shape of the regions in the neighborhood of the crack tip coincide with the shape of the plastic zone in plastically deformed materials; while the size of these zones is governed by a thermally dependent variable, which is analogous to the yield stress in plasticity.

Next, the transformation toughening behavior of shape memory alloys for mode I deformation was numerically obtained by means of resistance curves. Crack propagation was modeled using a cohesive zone with linear interface elements and a bilinear traction-separation law. From the results presented here, it was observed that for a fixed cohesive energy $\Gamma_{c}$, the resistance curves were sensitive to the normalized maximum traction in the cohesive zone $t_{0} / \hat{\sigma}$. An increase of this ratio produced an increase in the steady state stress intensity factor $K_{s s}$. This results from a relatively large transformation zone and significant energy dissipation. Consequently, a higher applied load is needed for crack propagation.

The effect of a reverse transformation on the transformation toughening of the alloy was examined as well. It was seen that transformation toughening depends upon the amount of reverse transformation. Values of the steady state stress intensity factor for different values of $t_{0} / \hat{\sigma}$ decreased as a result of the reverse transformation which decreases the amount of energy dissipation in the wake. Consequently, the applied load that is required for crack propagation 
also decreased.

Finally, the effect of the moduli mismatch between the austenite and martensite phases on the transformation toughening of shape memory alloys was examined. It was found that this mismatch produced an increase in the steady state stress intensity factor; hence, from a fracture mechanics point of view, shape memory alloys that exhibit this mismatch are preferred.

As further work, the effect of mixed mode deformation on the transformation toughening of shape memory alloys may be examined. In this case, the crack does not necessarily propagate in the direction ahead of the crack tip, but in a direction that depends on the mode mixity. Hence, a different approach should be considered. Another option is to investigate the behavior of delaminations between a laminate composed of shape memory alloy fibers embedded in a polymer or metal matrix and an isotropic, homogeneous medium. Finally, the problem of shape memory alloy fiber debond may be studied and examined for different traction separation laws.

\section{A Time discrete constitutive model of SMA}

Computationally, the non-linear behavior of the model presented by Panoskaltsis et al. (2004) may be treated as an implicit time discrete strain driven problem. Accordingly, the time discrete equations of the model are integrated over a time interval $(t, t+\Delta t)$ using an implicit backward Euler scheme, which is unconditionally stable. During elastic deformation

$$
\begin{aligned}
\xi^{t+\Delta t} & =\xi^{t}, \\
\boldsymbol{\epsilon}^{t r, t+\Delta t} & =\boldsymbol{\epsilon}^{t r, t},
\end{aligned}
$$

and the new stress state is determined using eq. (10). For forward and reversed transformations, the discrete equations may be derived as

$$
\begin{aligned}
\xi^{t+\Delta t} & =\xi^{t}+\Delta \xi \\
\Delta \xi & =\frac{\mu_{\xi}^{t+\Delta t}}{\mu_{f}^{t+\Delta t}} \Delta F \\
\Delta F & =\frac{3}{2} \frac{J_{2}^{t+\Delta t}-J_{2}^{t}}{\bar{\sigma}^{t+\Delta t}}-c\left(T^{t+\Delta t}-T^{t}\right), \\
J_{2}^{t+\Delta t} & =\frac{1}{2} s^{t+\Delta t}: s^{t+\Delta t},
\end{aligned}
$$




$$
\begin{aligned}
\boldsymbol{s}^{t+\Delta t} & =\frac{E^{t+\Delta t}}{1+\nu^{t+\Delta t}}\left(\boldsymbol{\epsilon}^{t+\Delta t}-\frac{1}{3} \boldsymbol{\epsilon}^{t+\Delta t}: \boldsymbol{I}-\boldsymbol{\epsilon}^{t r, t+\Delta t}\right), \\
\boldsymbol{\epsilon}^{t r, t+\Delta t} & =\boldsymbol{\epsilon}^{t r, t}+\Delta \xi \epsilon_{L} \boldsymbol{n}^{t+\Delta t},
\end{aligned}
$$

where $\boldsymbol{I}$ is the identity matrix, the normal to the transformation surface $\boldsymbol{n}$ is given in eq. (6),

$$
\mu_{\xi}=1-\xi, \quad \mu_{f}=-F_{M_{f}}, \quad c=C_{M}
$$

for the case of a forward transformation, and

$$
\mu_{\xi}=\xi, \quad \mu_{f}=F_{A_{f}}, \quad c=C_{A},
$$

for a backward transformation. The increment of the martensite fraction $\Delta \xi$ is obtained by solving the nonlinear equation

$$
\Delta \xi-\frac{3}{2} \frac{\mu_{\xi}^{t+\Delta t}}{\mu_{f}^{t+\Delta t}} \frac{J_{2}^{t+\Delta t}-J_{2}^{t}}{\bar{\sigma}^{t+\Delta t}}-c\left(T^{t+\Delta t}-T^{t}\right)=0
$$

and the new stress state may be determined using eqs. (A.2) and (10).

\section{B Cohesive zone element}

For the interface element in Fig. 5, Alfano and Crisfield (2001) defined the parameter

$$
\bar{\gamma}(\tau)=\max _{0 \leq \tau^{\prime} \leq \tau} \gamma\left(\tau^{\prime}\right)
$$

where $\tau$ is a pseudo-time parameter associated with time steps in the finite element solution. It may be shown that eq. (38) may be rewritten as

$$
\gamma\left(\tau^{\prime}\right)=\left[\left(\frac{<\delta_{1}\left(\tau^{\prime}\right)>}{\delta_{01}}\right)^{\alpha}+\left(\frac{\left|\delta_{2}\left(\tau^{\prime}\right)\right|}{\delta_{02}}\right)^{\alpha}\right]^{1 / \alpha}-1
$$


with $<$. $>$ the McCauley bracket (see Appendix D of Alfano and Crisfield, 2001, for details). Substituting eq. (B.2) into eqs.(34), (36) and (37) yields a traction-separation law for mixed mode deformation as

$$
\mathbf{t}(\tau)=\left\{\begin{array}{cc}
\mathbf{K} \boldsymbol{\delta}(\tau) & \bar{\gamma}(\tau) \leq 0 \\
{[\mathbf{I}-\mathbf{D}(\tau) \mathbf{C}(\tau)] \mathbf{K} \boldsymbol{\delta}(\tau)} & \bar{\gamma}(\tau)>0
\end{array}\right.
$$

where

$$
\mathbf{K}=\left[\begin{array}{cc}
t_{01} / \delta_{01} & 0 \\
0 & t_{02} / \delta_{02}
\end{array}\right],
$$

I is the $2 \times 2$ identity matrix, $\boldsymbol{\delta}=\left[\delta_{1}, \delta_{2}\right]^{T}$, and $\mathbf{D}(\tau)=\operatorname{diag}\left[d_{i}(\tau)\right]$ with

$$
d_{i}(\tau)=\min \left\{1, \frac{\bar{\gamma}(\tau)}{1+\bar{\gamma}(\tau)} \frac{\delta_{c i}}{\delta_{c i}-\delta_{0 i}}\right\}
$$

It may be noted that a correction has been made in eq. (B.5) for eq. $(16)_{2}$ in Alfano and Crisfield (2001); instead of 'max' it is 'min'. The matrix $\mathbf{C}(\tau)$ is defined by

$$
\mathbf{C}(\tau)=\left[\begin{array}{cc}
h\left[\delta_{1}(\tau)\right] & 0 \\
0 & 1
\end{array}\right]
$$

with the Heaviside step function, $h[x]=1$ if $x \geq 0$ and 0 , otherwise, so that penetration is avoided.

Writing eq. (B.3) incrementally leads to the constitutive tangent matrix $\mathbf{K}_{t}$ which is given by

$$
\mathbf{K}_{t}=\left\{\begin{array}{cc}
\mathbf{K} & \bar{\gamma} \leq 0 \\
(\mathbf{I}-\mathbf{D C}) \mathbf{K}-\frac{1}{(1+\bar{\gamma})^{1+\alpha}} \mathbf{F K}\left(\tilde{\boldsymbol{\delta}} \otimes \tilde{\boldsymbol{\delta}}^{\alpha-1}\right) \mathbf{A} & \bar{\gamma}=\gamma>0 \\
(\mathbf{I}-\mathbf{D C}) \mathbf{K} & \gamma<\bar{\gamma}>0
\end{array}\right.
$$


with the further condition $\left(K_{t}\right)_{i j}=0, j=1,2$ if $d_{i}>1$. In eq. (B.7), $\mathbf{A}=$ $\operatorname{diag}\left[1 / \delta_{0 i}^{\alpha}\right], \mathbf{F}$ is a diagonal matrix with the components $F_{i i}=\delta_{c i} /\left(\delta_{c i}-\delta_{0 i}\right)$,

$$
\tilde{\boldsymbol{\delta}}=\left\{\begin{array}{c}
<\delta_{1}> \\
\left|\delta_{2}\right|
\end{array}\right\} .
$$

and $\mathbf{a} \otimes \mathbf{b}$ is equivalent to $\mathbf{a} \cdot \mathbf{b}^{T}$ where $\mathbf{a}$ and $\mathbf{b}$ are vectors.

\section{References}

Alfano, G., Crisfield, M.A., 2001. Finite element interface models for the delamination analysis of laminated composites: mechanical and computational issues. International Journal for Numerical Methods in Engineering 50(7), 1701-1736.

Anderson, T.L., 2005. Fracture Mechanics: Fundamentals and Applications, CRC Press, Boca Raton, FL.

Auricchio, F., Petrini, L., 2002. Improvements and algorithmical considerations on a recent three-dimensional model describing stress-induced solid phase transformations. International Journal for Numerical Methods in Engineering 55(11), 1255-1284.

Auricchio, F., Petrini, L., 2004a. A three-dimensional model describing stresstemperature induced solid phase transformations: solution algorithm and boundary value problems. International Journal for Numerical Methods in Engineering 61(5), 807-836.

Auricchio, F., Petrini, L., 2004b. A three-dimensional model describing stresstemperature induced solid phase transformations: thermomechanical coupling and hybrid composite applications. International Journal for Numerical Methods in Engineering 61(6), 716-737.

Barenblatt, G.I., 1959. The formation of equilibrium cracks during brittle fracture. General ideas and hypotheses. Axially-symmetric cracks. Journal of Applied Mathematics and Mechanics (PMM) 23(3), 622-636.

Barenblatt, G.I., 1962. Mathematical theory of equilibrium cracks in brittle fracture. Advances in Applied Mechanics 7, 55-129.

Bathe, K.J., 2006. ADINA - Automatic Dynamic Incremental Nonlinear Analysis, Version 8.3.1.

Boyd, J.G., Lagoudas, D.C., 1996a. A thermodynamical constitutive model for shape memory alloys. Part I. The monolithic shape memory alloy. International Journal of Plasticity 12(6), 805-842.

Boyd, J.G., Lagoudas, D.C., 1996b. A thermodynamical constitutive model for 
shape memory alloys. Part II. The SMA composite material. International Journal of Plasticity 12(7), 843-873.

Budiansky, B., Hutchinson, J.W., Lambropoulis, J.C., 1983. Continuum theory of dilatant transformation toughening in ceramics. International Journal of Solids and Structures 19(4), 337-355.

Dugdale, D.S., 1960. Yielding of steel sheets containing slits. Journal of the Mechanics and Physics of Solids 8(2), 100-104.

Evans, A.G., 1984. Toughening mechanism in zirconia alloys. Advanced Ceramics 12(1), 193-212.

Geubelle, P.H., Baylor, J., 1998. Impact-induced delamination of laminated composites: a 2D simulation. Composites Part B: Engineering 29(5), 589602 .

Hutchinson, J.W., 1974. On steady state quasi-static crack growth. Harvard University Report, Division of Applied Sciences, DEAP S-8.

Janke, L., Czaderski, C., Motavalli, M., Ruth, J., 2005. Applications of shape memory alloys in civil engineering structures - overview, limits and new ideas. Materials and Structures 38(279), 578-592.

Li, H., Chandra, N., 2003. Analysis of crack growth and crack-tip plasticity in ductile materials using cohesive zone models. International Journal of Plasticity 19(6), 849-882.

Lu, Z.K., Weng, G.J., 1997. Martensitic transformation and stress-strain relations of shape-memory alloys. Journal of the Mechanics and Physics of Solids 45(11-12), 1905-1928.

Luo, Y., Salvia, M., 2004. SMA actuators in medical applications. Annales De Chimie-Science Des Materiaux 29(6), 33-41.

McKelvey, A.L., Ritchie, R.O., 2001. Fatigue-crack growth behavior in the superelastic and shape-memory alloy Nitinol. Metallurgical and Materials Transactions A-Physical Metallurgy and Materials Science 32(3A), 731-743.

McMeeking, R.M., Evans, A.G., 1982. Mechanics of transformationtoughening in brittle materials. Journal of the American Ceramic Society 65(5), 242-246.

Morgan, N.B., 2004. Medical shape memory alloy applications - the market and its products. Materials Science and Engineering: A - Structural Materials: Properties, Microstructure and Processing 378(1-2), 16-23.

Needleman, A., 1987. A continuum model for void nucleation by inclusion debonding. Journal of Applied Mechanics 54(3), 525-531.

Panoskaltsis, V.P., Bahuguna, S., Soldatos, D., 2004. On the thermomechanical modeling of shape memory alloys. International Journal of Non-Linear Mechanics 39(5), 709-722.

Patoor, E., Eberhardt, A., Berveiller, M., 1996. Micromechanical modelling of superelasticity in shape memory alloys. Journal de Physique IV C1-6, 277-292.

Patoor, E., Lagoudas, D.C., Entchev, P.B., Brinson, L.C., Gao, X.J., 2006. Shape memory alloys, Part I: General properties and modeling of single crystals. Mechanics of Materials 38(5-6), 391-429. 
Razov, A.I., 2004. Application of titanium nickelide-based alloys in engineering. Physics of Metals and Metallography 97(1), S97-S126.

Ritchie, R.O., 1999. Mechanisms of fatigue-crack propagation in ductile and brittle solids. International Journal of Fracture 100(1), 55-83.

Robertson, S.W., Ritchie, R.O., 2007. In vitro fatigue-crack growth and fracture toughness behavior of thin-walled superelastic Nitinol tube for endovascular stents: A basis for defining the effect of crack-like defects. Biomaterials 28(4), 700-709.

Schellekens, H., De Borst, R., 1993. On the numerical integration of interface elements. International Journal for Numerical Methods in Engineering 36(9), 43-66.

Song, G., Ma, N., Li, H.-N., 2006. Applications of shape memory alloys in civil structures. Engineering Structures 28(9), 1266-1274.

Souza, A.C., Mamiya, E.N., Zouain, N., 1998. Three-dimensional model for solids undergoing stress-induced phase transformations. European Journal of Mechanics, A: Solids 17(5), 789-806.

Stam, G., van der Giessen, E., 1995. Effect of reversible phase transformations on crack growth. Mechanics of Materials 21(1), 51-71.

Sun, Q.P., Hwang, K.C., Yu, S.U., 1991. A micromechanics constitutive model of transformation plasticity with shear and dilatation effect. Journal of the Mechanics and Physics of Solids 39(4), 507-524.

Sun, Q.P., Hwang, K.C., 1993a. Micromechanics modelling for the constitutive behavior of polycrystalline shape memory alloys. 1. Derivation of general relations. Journal of the Mechanics and Physics of Solids 41(1), 1-17.

Sun, Q.P., Hwang, K.C., 1993b. Micromechanics modelling for the constitutive behavior of polycrystalline shape memory alloys. 2. Study of the individual phenomena. Journal of the Mechanics and Physics of Solids 41(1), 19-33.

Tvergaard, V., Hutchinson, J.W., 1992. The relation between crack growth resistance and fracture process parameters in elastic-plastic solids. Journal of the Mechanics and Physics of Solids 40(6), 1377-1397.

Tvergaard, V., Hutchinson, J.W., 1993. The influence of plasticity on mixed mode interface toughness. Journal of the Mechanics and Physics of Solids 41(6), 1119-1135.

van den Bosch, M.J., Schreurs, P.J.G., Geers, M.G.D., 2006. An improved description of the exponential $\mathrm{Xu}$ and Needleman cohesive zone law for mixed-mode decohesion. Engineering Fracture Mechanics 73(9), 1220-1234.

Wilkes, K.E., Liaw, P.K., 2000. The fatigue behavior of shape-memory alloys. JOM-Journal of the Minerals, Metals and Materials Society 52(10), 45-51.

Yan, W., Wang, C.H., Zhang, X.P., Mai, Y.W., 2002. Effect of transformation volume contraction on the toughness of superelastic shape memory alloys. Smart Materials and Structures 11(6), 947-955.

Yi, S., Gao, S., 2000. Fracture toughening mechanism of shape memory alloys due to martensite transformation. International Journal of Solids and Structures 37(38), 5315-5327.

Yi, S., Gao, S., Shen, L., 2001. Fracture toughening mechanism of shape mem- 
ory alloys under mixed-mode loading due to martensite transformation. International Journal of Solids and Structures 38(24-25), 4463-4476. 